\title{
Modified Simple Equation Method and its Applications for some Nonlinear Evolution Equations in Mathematical Physics
}

\author{
Elsayed M. E. Zayed \\ Mathematics department, Faculty of Science \\ Zagazig University, Zagazig, Egypt
}

\author{
Hoda Ibrahim S. A. \\ Mathematics department, Faculty of Science \\ Zagazig University, Zagazig, Egypt
}

\begin{abstract}
In this paper, we employ the modified simple equation method to find the exact traveling wave solutions involving parameters of nonlinear evolution equations via the $(1+1)$ dimensional generalized shallow water-wave equation and the(2+1)-dimensional KdV-Burgers equation. When these parameters are taken to be special values, the solitary wave solutions are derived from the exact traveling wave solutions. It is shown that the proposed method provides a more powerful mathematical tool for constructing exact traveling wave solutions for many other nonlinear evolution equations.
\end{abstract}

PACS: 02.30.Jr, 05.45.Yv, 02.30.Ik.

\section{Keywords}

Modified simple equation method; Nonlinear evolution equations; Exact traveling wave solutions; Solitary wave solutions. .

\section{INTRODUCTION}

Nonlinear phenomena come out in a broad range of scientific applications, such as the fluid dynamics, nuclear physics, high energy physics, plasma physics, solid state physics, optical fibers, biology, chemical kinematics, chemical physics and so on. Mathematical modeling of many physics system leads to nonlinear evolution equations in various fields of science and engineering. Because of the increased concentration in the theory of solitary waves, a large variety of analytic and computational methods have been established in the analysis of the nonlinear models. For example the inverse scattering transformation method [1], the Hirota bilinear transform method [2], the Painleve integration method [3-6], the Backlund transformation method [7,8], the exp-function method [9-13], the tanh-function method [1417], the Jacobi-elliptic function expantion method [18-20], the $\left(G^{\prime} / G\right)$-expansion method [21-29], the $\left(G^{\prime} / G, 1 / G\right)$-expansion method [30,31], the first integral method [32], the variational iteration method [33], the homotopy perterbation method [34], the modified simple equation method [35-39] and so on. Recently, Jawad et al [35], Zayed [36] and Zayed et al [3739] have employed the modified simple equation method and found the exact traveling wave solutions of some nonlinear evolution equations via the Fitzhugh-Nagumo equation, the Sharma- Tasso- Olver equation, the modified KdV equation, the reaction-diffusion equation and the KolmogorovPetrovskii- Piskunov equation. The objective of this paper is to apply the modified simple equation method to seek the exact traveling wave solutions and then the solitary wave solutions of some other nonlinear evolution equations which play an important role in mathematical physics via the $(1+1)$ dimensional generalized shallow water-wave equation and the $(2+1)$-dimensional $\mathrm{KdV}$-Burgers equation.This paper is organized as follows: In Sec. 2, the description of the modified simple equation method is given. In Sec. 3, the applications of this method to two nonlinear equations indicated above are obtained. In Sec.4, some conclusions are given.

\section{Description of the modified simple equation method}

Consider a nonlinear evolution equation in the form:

$F\left(u, u_{t}, u_{x}, u_{y}, u_{t t}, u_{x y}, \ldots\right)=0$,

where $F$ is a polynomial in $u(x, y, t)$ and its partial derivatives in which the highest derivatives and nonlinear terms are involved. In the following, we give the main steps of this method [35-39]:

Step1. Using the wave transformation

$u(x, y, t)=u(\xi), \quad \xi=x+y+t$,

to reduce Eq.(2.1) to the following ODE :

$P\left(\mathrm{u}, \mathrm{u}^{\prime}, \mathrm{u} ", \mathrm{u}^{\prime \prime}, \ldots ..\right)=0$,

where $P$ is a polynomial in $u(\xi)$ and its total derivatives with respect to $\xi$.

Step 2. We suppose that Eq.(2.3) has the formal solution

$u(\xi)=\sum_{k=0}^{N} A_{k}\left(\frac{\psi^{\prime}(\xi)}{\psi(\xi)}\right)^{\mathrm{k}}$,

where $A_{k}$ are constants to be determined, such that $A_{N} \neq 0$. The function $\psi(\xi)$ is an unknown function to be determined later, such that $\psi^{\prime}(\xi) \neq 0$.

Step 3 . We determine the positive integer $N$ in (2.4) by balancing the highest order derivatives and the nonlinear terms in Eq. (2.3).

Step 4. We substitute (2.4) into Eq.(2.3) and calculate all the necessary derivatives $u^{\prime}, u^{\prime \prime}, u^{\prime \prime \prime}, \ldots$ of the unknown function $u(\xi)$ and we account the function $\psi(\xi)$. As a result of this substitution, we obtain a polynomial of $\left(\frac{\psi r(\xi)}{\psi(\xi)}\right)$ and its derivatives. In this polynomial, we gather all the terms of the 
same power of $\left(\frac{\psi \prime(\xi)}{\psi(\xi)}\right)$ and its derivatives, and we equate with zero all the coefficients of this polynomial. This operation yields a system of equations which can be solved without using the computer programs to find $A_{k}$ and $\psi(\xi)$. Consequently, we can obtain the exact solutions of Eq.(2.1).

\section{Applications}

In this section, we will apply the proposed method of Sec. 2, to find the exact solutions and then the solitary wave solutions of the following nonlinear evolution equations:

\subsection{Example 1. The generalized shallow water-wave equation}

This equation is well known [23, 40, 41] and has the form:

$u_{x x x t}+\alpha u_{x} u_{x t}+\beta u_{t} u_{x x}-u_{x t}-\gamma u_{x x}=0$

where $\alpha, \beta$ and $\gamma$ are nonzero constants. This equation can be derived from the classical shallow water theory in the socalled Boussinesq approximation [41]. The solution of Eq.(3.1) has been investigated by using other methods, namely, the improved Jacobi-elliptic function method [40], and the $\left(G^{\prime} / G\right)$-expansion method [23]. Let us now investigate Eq.(3.1) using the modified simple equation method. To this end, we use the wave trans- formation

$$
u(x, t)=u(\xi), \quad \xi=x-t,
$$

to reduce Eq.(3.1) into the following ODE:

$-u^{\prime \prime \prime}-\frac{1}{2}(\alpha+\beta) u^{\prime 2}+(1-\gamma) u^{\prime}=0$,

with zero constant of integration. Balancing $u^{\prime \prime \prime}$ with $u^{\prime 2}$ yields $N=1$. Consequently, we get

$u(\xi)=\mathrm{A}_{0}+\mathrm{A}_{1}\left(\frac{\psi^{\prime}(\xi)}{\psi(\xi)}\right)$,

where $A_{0}$ and $A_{1}$ are constants to be determined later, such as $\mathrm{A}_{1} \neq 0$. The function $\psi(\xi)$ is also to be determined, such that $\psi^{\prime}(\xi) \neq 0$. It is easy to see that

$u^{\prime}=\mathrm{A}_{1}\left(\frac{\psi^{\prime \prime}}{\psi}-\frac{\psi^{\prime 2}}{\psi^{2}}\right)$,

$$
\begin{aligned}
\boldsymbol{u}^{\prime \prime} & =\mathbf{A}_{\mathbf{1}}\left(\frac{\psi^{\prime \prime \prime}}{\boldsymbol{\psi}}-3 \frac{\psi^{\prime} \boldsymbol{\psi}^{\prime \prime}}{\boldsymbol{\psi}^{2}}+2 \frac{\psi^{\prime 3}}{\boldsymbol{\psi}^{3}}\right), \\
u^{\prime \prime \prime} & =\mathrm{A}_{1}\left(\begin{array}{c}
\frac{\psi^{\prime \prime \prime \prime}}{\psi}-4 \frac{\psi^{\prime} \psi^{\prime \prime \prime}}{\psi^{2}}-3 \frac{\psi^{\prime \prime 2}}{\psi^{2}}+ \\
12 \frac{\psi^{\prime 2} \psi^{\prime \prime}}{\psi^{3}}-6 \frac{\psi^{\prime 4}}{\psi^{4}}
\end{array}\right) .
\end{aligned}
$$

Substituting (3.5) and (3.7) into (3.3) and equating all the coefficients of $\psi^{-1}, \psi^{-2}, \psi^{-3}$ and $\psi^{-4}$ to be zero, we respectively obtain

$-\boldsymbol{\psi}^{\prime \prime \prime \prime}+(1-\gamma) \boldsymbol{\psi}^{\prime \prime}=0$,

$4 A_{1} \psi^{\prime} \psi^{\prime \prime \prime}+\psi^{\prime \prime 2}\left[3 \mathrm{~A}_{1}-\frac{1}{2}(\alpha+\beta) \mathrm{A}_{1}^{2}\right]-$ $(1-\gamma) \mathrm{A}_{1} \quad \psi^{\prime 2}=0$,

$A_{1} \quad \psi^{\prime 2} \psi^{\prime \prime}\left[(\alpha+\beta) A_{1}-12\right]=0$,

$A_{1} \quad \psi^{\prime 4}\left[12-(\alpha+\beta) A_{1}\right]=0$.

Since $\mathrm{A}_{1} \neq 0$ and $\psi^{\prime}(\xi) \neq 0$, we deduce from Eqs.(3.10) or (3.11) that $\mathrm{A}_{1}=\frac{12}{(\alpha+\beta)}, \alpha+\beta \neq 0$. Consequently, Eqs.(3.8) and (3.9) reduce to

$-\psi^{\prime \prime \prime \prime}+(1-\gamma) \psi^{\prime \prime}=0$

$4 \psi^{\prime} \psi^{\prime \prime \prime}-3 \psi^{\prime \prime 2}-(1-\gamma) \psi^{\prime 2}=0$

Integrating Eq.(3.12) and using Eq.(3.13) we get

$\frac{\psi^{\prime \prime \prime}}{\psi^{\prime \prime}}= \pm \sqrt{1-\gamma}$

with zero constant of integration, and $\gamma \neq 1$. Consequently, we deduce that

$\psi^{\prime}= \pm \frac{\mathrm{C}_{1}}{\sqrt{1-\gamma}} \exp ( \pm \xi \sqrt{1-\gamma})$

$\psi=C_{2} \pm \frac{\mathrm{C}_{1}}{(1-\gamma)} \exp ( \pm \xi \sqrt{1-\gamma})$

where $\mathrm{C}_{1}$ and $C_{2}$ are constants of integration. Now, the exact solution of Eq.(3.1) has the form:

$$
u(x, t)=A_{0} \pm \frac{12 C_{1} \sqrt{1-\gamma}}{(\alpha+\beta)}\left\{\frac{\exp ( \pm \sqrt{1-\gamma}(x-t))}{C_{2}(1-\gamma)+C_{1} \exp ( \pm \sqrt{1-\gamma}(x-t))}\right\}
$$

where $A_{0}$ is an arbitrary constant. If we set $C_{1}=1$ and $C_{2}=$ $\frac{1}{(1-\gamma)}$ in Eq.(3.17), where $\gamma \neq 1$, then we have the following solitary wave solutions:

$$
\begin{array}{r}
u_{1,2}(x, t)=A_{0} \pm \frac{6 \sqrt{1-\gamma}}{(\alpha+\beta)} \\
\left.\tanh \left[\frac{1}{2} \sqrt{1-\gamma}(x-t)\right]\right\},
\end{array}
$$

while, if $C_{1}=-1$ and $C_{2}=\frac{1}{(1-\gamma)}$, we have the solitary wave solutions:

$$
\begin{gathered}
u_{3,4}(x, t)=A_{0} \pm \frac{6 \sqrt{1-\gamma}}{(\alpha+\beta)}\{1 \pm \\
\left.\operatorname{coth}\left[\frac{1}{2} \sqrt{1-\gamma}(x-t)\right]\right\} .
\end{gathered}
$$




\subsection{Example 2. The KdV-Burgers equation}

This equation is well known $[28,33,34]$ and has the form:

$\left(u_{t}+u u_{x}-q u_{x x}+\mu u_{x x x}\right)_{x}+r u_{y y}=0$,

where $q, \mu$ and $r$ are nonzero real parameters. Eq.(3.20) is a wide class of nonlinear wave models of fluid in an elastic tube, liquid with small bubbles and turbulence. The solution of Eq. (3.20) has been investigated by using other methods via the modified variational iteration method [33], the variational homotopy perturbation method [34] and the $\left(G^{\prime} / G\right)$-expansion method [28]. Let us now solve Eq.(3.20) using the modified simple equation method. To this end, we use the wave transformation (2.2) to reduce Eq.(3.20) into the following ODE:

$(r+1) u+\frac{1}{2} u^{2}-q u^{\prime}+\mu u^{\prime \prime}=0$,

with zero constants of integration. Balancing $u^{\prime \prime}$ with $u^{2}$ in Eq.(3.21) yields $N=2$. Consequently, we have

$u(\xi)=\mathrm{A}_{0}+\mathrm{A}_{1}\left(\frac{\psi^{\prime}(\xi)}{\psi(\xi)}\right)+\mathrm{A}_{2}\left(\frac{\psi^{\prime}(\xi)}{\psi(\xi)}\right)^{2}$,

where $A_{0}, A_{1}$ and $A_{2}$ are constants to be determined, such that $A_{2} \neq 0$. Also, $\psi(\xi)$ can be determined such that $\psi^{\prime}(\xi) \neq$ 0 . It is easy to see that

$$
\begin{aligned}
u^{\prime}= & \mathrm{A}_{1}\left(\frac{\psi^{\prime \prime}}{\psi}-\frac{\psi^{\prime 2}}{\psi^{2}}\right)+2 \mathrm{~A}_{2}\left(\frac{\psi^{\prime} \psi^{\prime \prime}}{\psi^{2}}-\frac{\psi^{\prime 3}}{\psi^{3}}\right), \\
u^{\prime \prime}= & \mathrm{A}_{1}\left(\frac{\psi^{\prime \prime \prime}}{\psi}-3 \frac{\psi^{\prime} \psi^{\prime \prime}}{\psi^{2}}+2 \frac{\psi^{\prime 3}}{\psi^{3}}\right)+ \\
& \mathrm{A}_{2}\left(\frac{\psi^{\prime} \psi^{\prime \prime \prime}}{\psi^{2}}+\frac{\psi^{\prime \prime 2}}{\psi^{2}}-5 \frac{\psi^{\prime 2} \psi^{\prime \prime}}{\psi^{3}}+3 \frac{\psi^{\prime 4}}{\psi^{4}}\right) .
\end{aligned}
$$

Substituting (3.22)-(3.24) into (3.21) and equating all the coefficients of $\psi^{0}, \psi^{-1}, \psi^{-2}, \psi^{-3}$ and $\psi^{-4}$ to be zero, we respectively obtain

$(r+1) \mathrm{A}_{0}+\frac{1}{2} \mathrm{~A}_{0}^{2}=0$,

$$
\begin{aligned}
& (r+1) \mathrm{A}_{1} \psi^{\prime}+\mathrm{A}_{0} \mathrm{~A}_{1} \psi^{\prime}-\mathrm{qA}_{1} \psi^{\prime \prime}+\mu \mathrm{A}_{1} \psi^{\prime \prime \prime}=0,(3.26) \\
& (r+1) \mathrm{A}_{2} \psi^{\prime 2}+\frac{1}{2} \mathrm{~A}_{1}{ }^{2} \psi^{\prime 2}+\mathrm{A}_{0} \mathrm{~A}_{2} \psi^{\prime 2}+q \mathrm{~A}_{1} \psi^{\prime 2} \\
& \quad-2 q \mathrm{~A}_{2} \psi^{\prime} \psi^{\prime \prime}-3 \mu \mathrm{A}_{1} \psi^{\prime} \psi^{\prime \prime}+2 \mu \mathrm{A}_{2}\left(\psi^{\prime} \psi^{\prime \prime \prime}+\psi^{\prime 2}\right)=0,
\end{aligned}
$$

$$
\mathrm{A}_{1} \mathrm{~A}_{2} \psi^{\prime 3}+2 q \mathrm{~A}_{2} \psi^{\prime 3}+2 \mu \mathrm{A}_{1} \psi^{\prime 3}-10 \mu \mathrm{A}_{2} \psi^{\prime 2} \psi^{\prime \prime}=0,
$$

$\frac{1}{2} \mathrm{~A}_{2}{ }^{2} \psi^{\prime 4}+6 \mu \mathrm{A}_{2} \psi^{\prime 4}=0$.

Since $A_{2} \neq 0$ and $\psi^{\prime}(\xi) \neq 0$ we deduce from Eqs.(3.25) and (3.29) that

$\mathrm{A}_{0}=0, \mathrm{~A}_{0}=-2(r+1), \mathrm{A}_{2}=-12 \mu$,

where $r \neq-1$ and $\mu \neq 0$. Let us now discuss the following cases:

Case 1. If $\mathrm{A}_{0}=0, \mathrm{~A}_{1}=1$, then Eqs.(3.27) and (3.28) reduces to

$(r+1) \psi^{\prime 2}-2 \mathrm{q} \psi^{\prime} \psi^{\prime \prime}+2 \mu \psi^{\prime} \psi^{\prime \prime \prime}+2 \mu \psi^{\prime \prime 2}=0$,

$\psi^{\prime}=\frac{5 \mu}{\mathrm{q}} \psi^{\prime \prime}$.

Substituting (3.32) into (3.31) we get

$\frac{\psi^{\prime \prime \prime}}{\psi^{\prime \prime}}=\frac{A}{10 \mu q}$,

where $A=8 q^{2}-25 \mu(r+1) \neq 0$. Integrating (3.33) and using (3.32) we conclude that

$$
\begin{aligned}
\psi^{\prime} & =\frac{5 \mu \mathrm{C}_{1}}{\mathrm{q}} \exp \left(\frac{A \xi}{10 \mu q}\right), \\
\psi & =C_{2}+\frac{50 \mu^{2} \mathrm{C}_{1}}{\mathrm{~A}} \exp \left(\frac{A \xi}{10 \mu q}\right),
\end{aligned}
$$

where $C_{1}$ and $C_{2}$ are constants of integration.

Now, the exact solution of Eq. (3.20) in this case has the form

$$
u(x, y, t)=\frac{-300 C_{1}{ }^{2} \mu^{3}}{q^{2}}\left\{\frac{\exp \left[\frac{A}{10 \mu q}(x+y+t)\right]}{c_{2}+\frac{50 \mu^{2} C_{1}}{A} \exp \left[\frac{A}{10 \mu q}(x+y+t)\right]}\right\}^{2},
$$

If we set $C_{2}= \pm 1$ and $C_{1}=\frac{A}{50 \mu 2}$ into (3.36) then we have respectively the following solitary wave solutions:

$$
\begin{aligned}
& u_{1}(x, y, t)=\frac{-3 A^{2}}{100 \mu q^{2}}\left\{1+\tanh \left[\frac{A}{20 \mu q}(x+y+t)\right]\right\}^{2}, \\
& u_{2}(x, y, t)=\frac{-3 A^{2}}{100 \mu q^{2}}\left\{1+\operatorname{coth}\left[\frac{A}{20 \mu q}(x+y+t)\right]\right\}^{2},
\end{aligned}
$$

Case 2. If $A_{0}=0, A_{1} \neq 1$, then Eqs.(3.26), (3.27) and (3.28) reduce to

$$
(r+1) \psi^{\prime}-\mathrm{q} \psi^{\prime \prime}+\mu \psi^{\prime \prime \prime}=0 \text {, }
$$

$$
\begin{aligned}
& \psi^{2}\left[A_{1}^{2}+2 q A_{1}-24 \mu(r+1)\right]+\psi^{\prime} \psi^{\prime \prime}[48 q \mu-6 \mu \\
& -48 \mu^{2}\left[\psi^{\prime \prime 2}+\psi^{\prime} \psi^{\prime \prime}\right]=0 \\
& \psi^{\prime 2}\left[\left(5 A_{1}+12 q\right) \psi^{\prime}-60 \mu \psi^{\prime \prime}\right]=0
\end{aligned}
$$


Since $\psi^{\prime}(\xi) \neq 0$, we deduce from Eq.(3.41) that

$\psi^{\prime}=B \psi^{\prime \prime}$,

Where

$B=\frac{60 \mu}{5 A_{1}+12 q}$.

Substituting (3.42) into (3.40) we get

$\frac{\psi^{\prime \prime \prime}}{\psi^{\prime \prime}}=\frac{E}{48 q \mu^{2}}$,

where

$E=B^{2}\left(A_{1}^{2}+2 q A_{1}-24 \mu(r+1)+\right.$

$B 48 q \mu-6 \mu A 1-48 \mu 2$.

Consequently, we conclude that
$\boldsymbol{\Psi}^{\prime}=\mathrm{B} \boldsymbol{\Psi}^{\prime \prime}=\mathrm{C}_{\mathbf{1}} \mathrm{B} \exp \left(\frac{\mathrm{E} \xi}{48 \mu^{2} \mathrm{~B}}\right)$

(3.48)

$\psi=C_{2}+\frac{48 \mu^{2} B^{2} C_{1}}{E} \exp \left(\frac{E \xi}{48 \mu^{2} B}\right)$,

where $C_{1}$ and $C_{2}$ are constants of integration. Substituting (3.46)- (3.48) into (3.39) we deduce after some reduction that $A_{1}$ has the form:

$A_{1}=\frac{12}{5}\left[q \pm \sqrt{3 q^{2}-25 \mu(r+1)}\right]$.

Now, the exact solution of Eq.(3.20) in this case has the form:

$$
u(x, y, t)=A_{1} C_{1} B\left\{\frac{\exp \left[\frac{E}{48 \mu^{2} B}(x+y+t)\right]}{c_{2}+\frac{48 C_{1} \mu^{2} B^{2}}{E} \exp \left[\frac{E}{48 \mu^{2} B}(x+y+t)\right]}\right\}-12 \mu C_{1}{ }^{2} B^{2}\left\{\frac{\exp \left[\frac{E}{48 \mu^{2} B}(x+y+t)\right]}{c_{2}+\frac{48 C_{1} \mu^{2} B^{2}}{E} \exp \left[\frac{E}{48 \mu^{2} B}(x+y+t)\right]}\right\}^{2}
$$

If we set $C_{2} \pm 1$ and $C_{1}=\frac{E}{48 \mu^{2} B^{2}}$ into (3.51) then we have respectively the following solitary wave solutions

$u_{1}(x, y, t)=\frac{1}{2} A_{1} C_{1} B\left\{1+\tanh \left[\frac{E(x+y+t)}{96 \mu^{2} B}\right]\right\}-3 \mu C_{1}^{2} B^{2}\left\{1+\tanh \left[\frac{E(x+y+t)}{96 \mu^{2} B}\right]\right\}^{2}$,

$u_{2}(x, y, t)=\frac{1}{2} A_{1} C_{1} B\left\{1+\operatorname{coth}\left[\frac{E(x+y+t)}{96 \mu^{2} B}\right]\right\}-3 \mu C_{1}{ }^{2} B^{2}\left\{1+\operatorname{coth}\left[\frac{E(x+y+t)}{96 \mu^{2} B}\right]\right\}^{2}$.

Case3. If $\mathrm{A}_{0}=-2(r+1), \mathrm{A}_{1}=0$, then Eqs.(3.27) and (3.28) reduces to

$-(r+1) \psi^{\prime 2}-2 q \psi^{\prime} \psi^{\prime \prime}+2 \mu\left(\psi^{\prime} \psi^{\prime \prime}+\psi^{\prime \prime 2}\right)=0$, and

$$
u(x, y, t)=-2(r+1)-\frac{300 C_{1}^{2} \mu^{3}}{q^{2}}\left\{\frac{\exp \left[\frac{A}{10 \mu q}(x+y+t)\right]}{c_{2}+\frac{50 \mu^{2} C_{1}}{A} \exp \left[\frac{A}{10 \mu q}(x+y+t)\right]}\right\}^{2},
$$

while, the solitary wave solutions have the forms

$$
\begin{aligned}
& u_{1}(x, y, t)=-2(r+1)-\frac{3 A^{2}}{100 \mu q^{2}}\left\{1+\tanh \left[\frac{A}{20 \mu q}(x+y+t)\right]\right\}^{2}, \\
& u_{2}(x, y, t)=-2(r+1)-\frac{3 A^{2}}{100 \mu q^{2}}\left\{1+\operatorname{coth}\left[\frac{A}{20 \mu q}(x+y+t)\right]\right\}^{2},
\end{aligned}
$$

Case 4. If $A_{0}=-2(r+1), A_{1} \neq 0$,then Eqs. (3.26), (3.27) and (3.28) reduce to the same forms (3.39), (3.40) and (3.41) respectively with replacing $(r+1)$ by $-(r+1)$.
Thus the analysis and the solutions in this case follow directly from case 2 with replacing $(r+1)$ by $-(r+1)$. Now, the exact solution of Eq.(3.20) in this case has the for

$$
\begin{aligned}
& u(x, y, t)=-2(r+1)+A_{1} C_{1} B\left\{\frac{\exp \left[\frac{\mathrm{E}}{48 \mu^{2} \mathrm{~B}}(\mathrm{x}+\mathrm{y}+\mathrm{t})\right]}{\mathrm{c}_{2}+\frac{48 \mathrm{C}_{1} \mu^{2} \mathrm{~B}^{2}}{\mathrm{E}} \exp \left[\frac{\mathrm{E}}{48 \mu^{2} \mathrm{~B}}(\mathrm{x}+\mathrm{y}+\mathrm{t})\right]}\right\} \\
& -12 \mu C_{1}{ }^{2} B^{2}\left\{\frac{\exp \left[\frac{\mathrm{E}}{48 \mu^{2} \mathrm{~B}}(\mathrm{x}+\mathrm{y}+\mathrm{t})\right]}{\mathrm{c}_{2}+\frac{48 \mathrm{C}_{1} \mu^{2} \mathrm{~B}^{2}}{\mathrm{E}} \exp \left[\frac{\mathrm{E}}{48 \mu^{2} \mathrm{~B}}(\mathrm{x}+\mathrm{y}+\mathrm{t})\right]}\right\}^{2},
\end{aligned}
$$

while, the solitary wave solutions have the forms: 


$$
\begin{aligned}
& u_{1}(x, y, t)=-2(r+1)+\frac{1}{2} A_{1} C_{1} B\left\{1+\tanh \left[\frac{\mathrm{E}(\mathrm{x}+\mathrm{y}+\mathrm{t})}{96 \mu^{2} \mathrm{~B}}\right]\right\}-3 \mu C_{1}{ }^{2} B^{2}\left\{1+\tanh \left[\frac{\mathrm{E}(\mathrm{x}+\mathrm{y}+\mathrm{t})}{96 \mu^{2} \mathrm{~B}}\right]\right\}^{2}, \\
& u_{2}(x, y, t)=-2(r+1)+\frac{1}{2} A_{1} C_{1} B\left\{1+\operatorname{coth}\left[\frac{\mathrm{E}(\mathrm{x}+\mathrm{y}+\mathrm{t})}{96 \mu^{2} \mathrm{~B}}\right]\right\}-3 \mu C_{1}{ }^{2} B^{2}\left\{1+\operatorname{coth}\left[\frac{\mathrm{E}(\mathrm{x}+\mathrm{y}+\mathrm{t})}{96 \mu^{2} \mathrm{~B}}\right]\right\}^{2} .
\end{aligned}
$$

\section{Conclusions}

The modified simple equation method has been applied in this paper to find the exact traveling wave solutions and then the solitary wave solutions of two nonlinear evolution equations, namely, the $(1+1)$-dimensional generalized shallow waterwave equation (3.1) and the (2+1)-dimensional KdV-Burgers equation (3.20). Comparing the presently proposed method with other methods, we can conclude that the modified simple equation method is much more simpler than these methods and can be applied to many other nonlinear evolution equations in mathematical physics.

\section{ACKNOWLEDGMENTS}

The authors wish to thank the referees for their interesting suggestions and comments to improve this article.

\section{REFERENCES}

[1] Ablowitz M.. J. and Clarkson P. A., Solitons, Nonlinear Evolution Equation and Inverse Scattering, Cambridge University press, New York, 1991.

[2] Hirota, R. Exact solutions of the $\mathrm{KdV}$ equation and multiple collisions of solitons , Phys. Rev. Lett.,27 (1971) 1192-1194.

[3] Weiss J., Tabor M. and Carnvalle G., The Painleve property for PDEs., J. Math. Phys. 24 (1983) 522-526.

[4] Kudryashov N. A., Exact soliton solutions of the generalized evolution equation of wave dynamics, J. Appl. Math. Mech. 52 (1988) 361-365.

[5] Kudryashov N. A., Exact soliton solutions of the generalized Kuramoto-Sivashinsky equation, Phys. Lett. A 147 (1990) 287-291.

[6] Kudryashov N. A., On types nonlinear nonintegrable differential equations with exact solutions, Phys. Lett. A 155 (1991) 269-275.

[7] Miura M. R., Backlund transformation. Springer, Berline, 1978.

[8] Rogers C. and Shadwick W. F., Backlund transformation, Academic Press, New York, 1982.

[9] He J. H. and Wu X. H., Exp-function method for nonlinear wave equations, Chaos, Solitons \& Fractals, 30 (2006) 700-708.

[10] Yusufoglu E., New solitary solutions for the MBBM equations using the exp- function method. Phys. Lett. A 372 (2008) 442-446.

[11] Zhang S., Application of the exp- function method to high dimensional evolution equations, Chaos, Solitons \& Fractals, 38 (2008) 270- 276.

[12] Bekir A., The exp-function method for Ostrovsky equation, Int. J. Nonlinear Sci. Num. Simul. 10 (2009) 735-739.
[13] Bekir A., Application of the exp-function method for nonlinear differential-difference equations, Appl. Math. . Comput., 215 (2010) 4049-4053.

[14] Abdou M. A., The extended tanth-method and its applications for solving nonlinear physical models, Appl. Math. Comput. 190 (2007) 988-996.

[15] Fan E. G., Extended tanh-function method and its applications to nonlinear equations, Phys. Lett. A 277 (2000) 212-218.

[16] Zhang S. and Xia T. C., A further improved tanhfunction method exactly solving (2+1)-dimensional dispersive long wave equations, Appl. Math. E-Notes, 8 (2008) 58-66.

[17] Yusufoglu E. and Bekir A., Exact solutions of coupled nonlinear Klein- Gordon equations, Math. Comput. Modeling, 48 (2008) 1694-1700.

[18] Chen Y. and Wang Q., Extended Jacobi elliptic function rational expansion method and abundant families of Jacobi elliptic function solutions to the(1+1)-dimensional nonlinear dispersive long wave equation. Chaos, Solitons \& Fractals. 24 (2005) 745-757.

[19] Liu S., Fu Z., Liu S. D. and Zhao Q., Jacobi elliptic function expansion method and periodic wave solutions of nonlinear equations. Phys. Lett. A. 289 (2001) 69-74.

[20] Lu D., Jacobi elliptic function solutions for two variant variant Boussineq equation, Chaos, Solitons. \& Fractals, 24 (2005) 1373-1375.

[21] Wang M. L., Li X. and Zhang J., The (G'/G) -expansion method and traveling wave solutions of nonlinear evolution equations in mathematical physics, Phys. Lett. A 372, 4 (2008) 417-423.

[22] Zhang S., Tong J. L. and Wang W. , A generalized $\left(G^{\prime} / G\right)$-expansion method for the KdV equation with variable coefficients, Phys. Lett. A 372 (2008) 22542257.

[23] Zayed E. M. E. and Gepreel K. A. The (G'/G) expansion method for finding traveling wave solutions of nonlinear partial differential equations in mathematical physics. J. Math. Phys. 50 (2009) 013502- 013513.

[24] Zayed E. M. E. , The (G'/G) -expansion method and its applications to some nonlinear evolution equations in mathematical physics. J. Appl. Math. Computing, 30 (2009) 89-103.

[25] Bekir A., Application of the $\left(\mathrm{G}^{\prime} / \mathrm{G}\right)$-expansion method for nonlinear evolution equations, Phys. Lett. A 372 (2008) 3400-3406.

[26] Ayhan B. and Bekir A.,The (G'/G) -expansion method for nonlinear lattice equations, Commu. Nonlinear Sci. Numer. Simula. 17 (2012) 3490-3498

[27] Kudryashov N. A., A note on the $\left(\mathrm{G}^{\prime} / \mathrm{G}\right)$-expansion method, Appl. Math. Comput. 217 (2010) 1755-1758. 
[28] Aslan I. and Ozis T., Analytic study on two nonlinear evolution equations by using the $\left(\mathrm{G}^{\prime} / \mathrm{G}\right)$-expansion method, Appl. Math. Comput. 209 (2009) 425-429.

[29] Kudryashov N. A., Meromorphic solutions of nonlinear ordinary differential equations, Comm. Nonlinear Sci. Numer. Simula. 15 (2010) 2778-2790.

[30] Li X. L., Li Q. E. and Wang L.W., The (G'/G)-expansion method and its application to traveling wave solutions of Zakharov equations, Appl. Math. J. Chinese Univ. 25 (2010) 454-462.

[31] Zayed E. M. E. and Abdelaziz M. A. M. , The two variable $\left(G^{\prime} / G, 1 / G\right)$-expansion method for solving the nonlinear KdV-mKdV equation, Math. Prob. Eng. Vol. 2012, Article ID 725061, 14 pages.

[32] Tascan F. and Bekier A., Applications of the first integral method to the nonlinear evolution equations, Chinese Phys. B 19 (2010) 080201-4.

[33] Zayed E. M. E. and Abdel Rahman H. M., On solving the KdV-Burgers equation and the $\mathrm{Wu}$ - Zhang equations using the modified variational iteration method, Int. J. Nonlinear Sci. Numer. Simula. 10 (2009) 1093-1103.

[34] Zayed E. M. E. and Abdel Rahman H. M., The variational iteration method and the variational homotopy perterbation method for solving the KdVBurgers equation and the Sharma-Tasso- Olver equation, Z. Naturforsch, 65a (2010) 25-33.
[35] Jawad A. T. M., Patkovic M. D. and Biswas A., Modified simple equation method for nonlinear evolution equation, Appl. Math. Compu., 217 (2010) 869-877.

[36] Zayed E. M. E., A note on the modified simple equation method applied to Sharma-Tasso- Olver equation, Appl. Math. Comput., 218 (2011) 3962-3964.

[37] Zayed E. M. E. and Hoda Ibrahim S. A., Exact solutions of nonlinear evolution equations in mathematical physics using the modified simple equation method, Chinese Phys, Lett. 29 (2012) 060201-060204.

[38] Zayed E. M. E. and Hoda Ibrahim S. A., Exact solutions of Kolmogorov-Petrovskii-Piskunov equation using the modified simple equation method, Acta Math. Appl. Sinica (English Series), Accepted.

[39] Zayed E. M. E. and Arnous A. H. , Exact solutions of the nonlinear ZK-MEW and the Potential YTSF equations using the modified simple equation method, AIP Conference Proceedings 1479 (2012) 2044-2048.

[40] Inc M. and Ergu M., Periodic wave solutions for the generalized shallow water-wave equation by the improved Jacobi- elliptic function method, Appl. Math. E-Notes, 5 (2005) 89-96.

[41] Whitham G. B., Linear and nonlinear waves, Wiley, New York, 1974. 